\title{
Commerson's Leaf-Nosed Bat (Hipposideros commersoni) is the Likely Reservoir of Shimoni Bat Virus
}

\author{
Ivan V. Kuzmin, Amy S. Turmelle, Bernard Agwanda, ${ }^{2}$ Wanda Markotter, ${ }^{3}$ Michael Niezgoda, \\ Robert F. Breiman, ${ }^{4}$ and Charles E. Rupprecht ${ }^{1}$
}

\begin{abstract}
In this study we attempted to identify whether Commerson's leaf-nosed bat (Hipposideros commersoni) is the reservoir of Shimoni bat virus (SHIBV), which was isolated from a bat of this species in 2009. An alternative explanation is that the isolation of SHIBV from H. commersoni was a result of spill-over infection from other species, particularly from the Egyptian fruit bats (Rousettus aegyptiacus), which frequently sympatrically roost with $H$. commersoni and are known as the reservoir of the phylogenetically related Lagos bat virus (LBV). To evaluate these hypotheses, 769 bats of at least 17 species were sampled from 18 locations across Kenya during 2009-2010. Serum samples were subjected to virus neutralization tests against SHIBV and LBV. A limited amount of cross-neutralization between LBV and SHIBV was detected. However, H. commersoni bats demonstrated greater seroprevalence to SHIBV than to LBV, and greater virus-neutralizing titers to SHIBV than to LBV, with a mean difference of $1.16 \log _{10}(95 \%$ confidence intervals [CI]: $0.94-1.40 ; p<0.001)$. The opposite pattern was observed for sera of $R$. aegyptiacus bats, with a mean titer difference of $1.06 \log _{10}$ (95\% CI: $0.83-1.30 ; p<0.001$ ). Moreover, the seroprevalence in $H$. commersoni to SHIBV in the cave where these bats sympatrically roosted with $R$. aegyptiacus (and where SHIBV was isolated in 2009) was similar to their seroprevalence to SHIBV in a distant cave where no $R$. aegyptiacus were present $(18.9 \%$ and $25.0 \%$, respectively). These findings suggest that H. commersoni is the host species of SHIBV. Additional surveillance is needed to better understand the ecology of this virus and the potential risks of infection to humans and other mammalian species.
\end{abstract}

Key Words: Bat—Field studies—Kenya—Lyssavirus—Rabies—Serology—Shimoni bat virus—SeroprevalenceSurveillance-Zoonosis.

\section{Introduction}

A T PRESENT, 11 SPECIES are recognized in the Lyssavirus genus (Family Rhabdoviridae, Order Mononegavirales), including Rabies virus (RABV), Lagos bat virus (LBV), Mokola virus (MOKV), Duvenhage virus (DUVV), European bat lyssaviruses type 1 and 2, Australian bat lyssavirus, Aravan virus, Khujand virus, Irkut virus, and West Caucasian bat virus (WCBV) (Dietzgen et al., in press). A new lyssavirus, named Shimoni bat virus (SHIBV), was isolated during 2009 in coastal Kenya from a freshly dead insectivorous bat Hipposideros commersoni (Kuzmin et al. 2010). Phylogenetically, SHIBV belongs to Phylogroup II lyssaviruses, which also includes MOKV and LBV (Badrane et al. 2001). However, based on genetic dis- tances and topology of phylogenetic trees, SHIBV could not be included into any of these prior lyssavirus species. Instead, SHIBV was proposed as a new species of Lyssavirus genus (Kuzmin et al. 2010).

Within the phylogroup II lyssaviruses, MOKV has never been documented in bats, and the reservoir host of MOKV is unknown. In contrast, the reservoir hosts of LBV are several species of African fruit bats (Family Pteropodidae) (Kuzmin et al. 2008a, Markotter et al. 2008). As only one isolate of SHIBV has been obtained to date, no conclusion could be made on the reservoir host of this virus. The system of three caves where SHIBV was encountered is inhabited by bats of at least eight species, including a large colony of Egyptian fruit bats (Rousettus aegyptiacus), which are recognized hosts of

\footnotetext{
${ }^{1}$ Rabies Program, Poxvirus and Rabies Branch, Division of High-Consequence Pathogens and Pathology, Centers for Disease Control and Prevention, Atlanta, Georgia.

${ }^{2}$ Mammalogy Section, National Museum of Kenya, Nairobi, Kenya.

${ }^{3}$ Department of Microbiology and Plant Pathology, Faculty of Natural and Agricultural Sciences, University of Pretoria, Pretoria, South Africa.

${ }^{4}$ Centers for Disease Control and Prevention in Kenya, Nairobi, Kenya.
} 
LBV (Aubert 1999, Kuzmin et al. 2008a, Markotter et al. 2008). As SHIBV belongs to the same viral phylogroup, a possibility existed that R. aegyptiacus is the reservoir host of SHIBV, and isolation of this virus from $H$. commersoni was due to an incidental spill-over infection.

Only a few $H$. commersoni bats were sampled in previous surveys of Kenya bats for lyssaviruses (Kuzmin et al. 2008a, 2008b). Additional difficulty is associated with serologic cross-reactivity of lyssaviruses, documented within phylogroups I and II (but not between the phylogroups) (Badrane et al. 2001). As the glycoprotein sequence of SHIBV is similar to that of LBV, serologic cross-reactivity between these viruses (and to a lesser extent with MOKV) was expected (Kuzmin et al. 2010). Therefore, simple serologic screening of bats for antibodies to SHIBV would not allow reliable identification of the host species. In the present study, we attempted to resolve this issue via extensive comparative survey of bats, collected in Kenya during 2009-2010, for their seroprevalence to SHIBV and LBV. We primarily focused on $H$. commersoni and $R$. aegyptiacus, but also collected other bat species at different locations across the country, including sites where crossspecies exposure within bat roosts would be unlikely.

The current taxonomy of the Hipposideros genus is not well developed, although 67 species are recognized (Simmons 2005). The identification of $H$. commersoni in our previous studies (Kuzmin et al. 2008a, 2010) was based on an early description (Kingdon 1974). Later, H. commersoni was subdivided into four species. Among these, H. commersoni is believed to be present only in Madagascar, whereas two other species $H$. vittatus and $H$. gigas inhabit continental Africa, and the fourth species $H$. thomensis was described from São Tomé Island. Nevertheless, taxonomy of this group has not been resolved to date, no significant studies were published, and all four delineated species are currently included into the H. commersoni group (Simmons 2005). Moreover, based on morphometric characters, McWilliam (1982) reported sympatric roosting of two species, $H$. commersoni and $H$. gigas, in caves of the Shimoni area of coastal Kenya. Given these discrepancies and the absence of reference genetic information from these species in the public domain, which could be used for comparison in our study, we refer to these bats as $H$. commersoni, awaiting further taxonomic clarifications.

\section{Materials and Methods}

\section{Bat sampling}

The study was performed in the framework of the Global Disease Detection Program, which focused on the detection of emerging infectious pathogens in Kenya bats. During 20092010, 769 bats of at least 17 species were collected from 18 locations across southern Kenya, from the western border (Mount Elgon) to the south-eastern coast (Fig. 1). Selection of sampling sites was based on available information about bat roosts and preliminary information on zoonotic pathogens detected in Kenya bats previously (for more information, see Kuzmin et al. 2008a, 2008b, 2010). The number of samples and collection protocol were approved by the National Museums of Kenya and Kenya Wildlife Service.

As special focus was given to SHIBV and the potential roles of H. commersoni and R. aegyptiacus as reservoirs, bats of these species were more extensively sampled. In addition to the cave where SHIBV was initially encountered, inhabited by

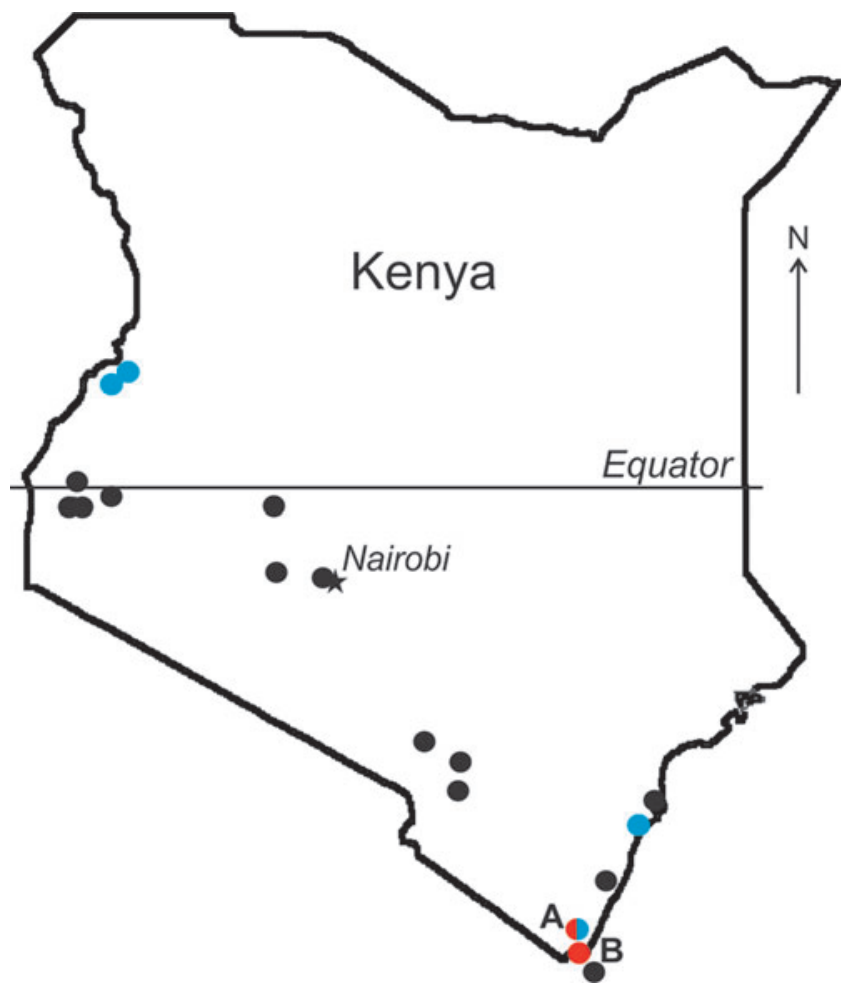

FIG. 1. Map of Kenya showing the locations of the bat collections (round marks). Red marks indicate sites of Hipposideros commersoni sampling, and blue marks indicate sites of Rousettus aegyptiacus sampling. A and B indicate two caves where $H$. commersoni were sampled, as described in the text. The star indicates the position of Nairobi.

bats of at least eight species, including R. aegyptiacus (Cave A), sampling of $H$. commersoni was also performed in a distant Cave $\mathrm{B}$, where only $H$. commersoni and a limited number of other insectivorous bats were found, roosting in different cave chambers, but $R$. aegyptiacus were not present. Similarly, $R$. aegyptiacus bats were sampled not only from Cave A, but also from several other caves where $H$. commersoni bats were not present.

Captured bats were anesthetized by an intramuscular injection of ketamine hydrochloride $(0.05-0.1 \mathrm{mg} / \mathrm{g}$ bodyweight) and sampled in compliance with the guidelines of the Institutional Animal Care and Use Committee of the Centers for Disease Control and Prevention. All bats were measured, sexed, and identified to species or genus level, using available field guides and genetic information obtained during the previous surveys in Kenya (Kuzmin et al. 2008a). The bats were euthanized under sedation, and various tissues and swabs were harvested for screening for different pathogens, which are out of the subject of this article. Bat carcasses were preserved in $10 \%$ buffered formalin for needs of the National Museums of Kenya. Blood samples were taken under anesthesia, and serum was separated by centrifugation. All samples were transported on dry ice and stored at $-80^{\circ} \mathrm{C}$ until use.

\section{Detection of virus-neutralizing antibodies}

The virus-neutralizing antibodies (VNA) were detected and measured by a modification of the rapid fluorescent focus 
inhibition test (RFFIT), as described elsewhere (Smith et al. 1996, Kuzmin et al. 2008a), using four-well (6 mm) tefloncoated glass slides (Cel-Line, Erie Scientific, Portsmouth, NH). Two viruses were used in the comparative reactions: SHIBV (original isolate, recovered from $H$. commersoni in Kenya during 2009) and LBV (isolate KE576, recovered from R. aegyptiacus in Kenya during 2008). For sustainable use in the RFFIT, both viruses were subjected to three passages in mouse neuroblastoma (MNA) cell culture. To ensure specificity of virus neutralization, and considering that Phylogroup II lyssaviruses do not cross-neutralize Phylogroup I lyssaviruses, we additionally tested all samples against DUVV (SouthAfrican isolate), a representative of Phylogroup I.

Initially, heat-inactivated bat serum samples were screened in dilutions 1:10 and 1:25. In brief, $3.5 \mu \mathrm{L}$ of serum was mixed in a well with $14 \mu \mathrm{L}$ Minimum Essential Medium (MEM-10). Further, $5 \mu \mathrm{L}$ of this mixture was transferred to another well and mixed with $7.5 \mu \mathrm{L}$ of MEM-10 (final volume in each well: $12.5 \mu \mathrm{L}$ ). Thereafter, $12.5 \mu \mathrm{L}$ of viral inocula was added into each well (virus dose varied from 28 to 80 focus forming units, as determined by titration in a control slide with each set of sera), and the slides were incubated in a humidity chamber for $90 \mathrm{~min}$ at $37^{\circ} \mathrm{C}$ in the presence of $0.5 \% \mathrm{CO}_{2}$. After incubation, MNA cells $\left(25 \mu \mathrm{L}\right.$ of $2 \times 10^{6}$ cells $\left./ \mathrm{mL}\right)$ were added into each well, and slides were incubated at the same conditions for $44 \mathrm{~h}$ before acetone fixation and staining (Smith et al. 1996). On microscopy, 10 separate fields were observed for each well. If a reduction or absence of fluorescence was observed, the serum sample was subjected to additional titration in fivefold dilutions from 1:10 to 1:1250. The 50\% end-point neutralizing titers were calculated by the method of Reed and Muench (Smith et al. 1996). Only the samples that had a 50\% end-point neutralizing titer greater than $1 \log 10$ (e.g., less than 5 of the 10 counted fields contained infected cells at serum dilution 1:10) were considered as positive. Previous trials with RABV VNA demonstrated that results obtained by this micromethod are comparable to those obtained by the classical test in chamber slides (Smith et al. 1996).

\section{Statistical analysis}

Pairwise comparisons of VNA titers against SHIBV and LBV in H. commersoni and R. aegyptiacus were performed using the nonparametric sign test, whereas species-level VNA seroprevalence estimates to SHIBV and LBV were compared using a chi-square test. The $95 \%$ confidence intervals (95\% CI) were determined for VNA seroprevalence, and $p$-values less than 0.05 were considered statistically significant. The analyses were performed using software package STATISTICA, version 10 (StatSoft, Tulsa, OK).

\section{Results}

The overall results of serologic testing of bats against SHIBV and LBV are presented in Table 1 . The greatest number of seropositive bats was represented by $H$. commersoni and pteropodid bats, particularly by the most extensively sampled R. aegypticaus. However, reactivity patterns were different for SHIBV and LBV (Table 2). For example, only 25\% (5 of 20) $H$. commersoni that neutralized SHIBV also neutralized LBV. Moreover, the neutralizing titer of the serum of each individual $H$. commersoni bat that neutralized both viruses was greater against SHIBV than against LBV (Fig. 2), with a mean
Table 1. Kenya Bats Serologically Tested for Shimoni Bat Virus and Lagos Bat Virus During 2009-2010

\begin{tabular}{lrcc}
\hline Bat taxa & Tested & $\begin{array}{c}\text { Seropositive to } \\
\text { SHIBV }(\%)\end{array}$ & $\begin{array}{c}\text { Seropositive } \\
\text { to LBV }(\%)\end{array}$ \\
\hline Hipposideros commersoni & 99 & $20(20.2)$ & $5(5.1)^{\mathrm{a}}$ \\
Hipposideros sp. & 22 & $1(4.5)$ & 0 \\
Triaenops persicus & 8 & 0 & 0 \\
Cardioderma cor & 3 & 0 & 0 \\
Chaerephon sp. & 81 & 0 & 0 \\
Coleura afra & 24 & 0 & 0 \\
Miniopterus sp. & 235 & $2(0.9)$ & $2(0.9)$ \\
Neoromicia sp. & 47 & 0 & 0 \\
Nycteris sp. & 13 & 0 & 0 \\
Otomops martiensseni & 35 & 0 & 0 \\
Pipistrellus sp. & 4 & $1(25.0)$ & 0 \\
Rhinolophus sp. & 41 & 0 & 0 \\
Scotoecus sp. & 3 & 0 & 0 \\
Scotophilus dingani & 10 & 0 & 0 \\
Rousettus aegyptiacus & 79 & $26(32.9)^{\mathrm{b}}$ & $37(46.8)$ \\
Eidolon helvum & 9 & $2(22.2)^{\mathrm{c}}$ & $4(44.4)$ \\
Epomophorus sp. & 56 & $1(1.8)^{\mathrm{c}}$ & $1(1.8)$ \\
\hline
\end{tabular}

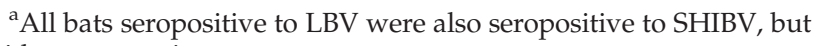
with a greater titer.

${ }^{b}$ All bats seropositive to SHIBV were also seropositive to LBV, and all but one with a greater titer.

${ }^{\mathrm{c}}$ All bats seropositive to SHIBV were also seropositive to LBV, but with a greater titer.

SHIBV, Shimoni bat virus; LBV, Lagos bat virus.

difference of $1.16 \log _{10}$ (95\% CI: 0.94-1.40, $Z=5.00, p<0.001$ ). In contrast, $70 \%$ (26 of 37 ) of R. aegyptiacus that demonstrated reactivity to LBV also neutralized SHIBV. The neutralizing titer of the serum of each individual $R$. aegyptiacus bat that neutralized both viruses was greater against LBV than against SHIBV (Fig. 2), with a mean difference of $1.06 \log _{10}(95 \%$ CI: $0.83-1.30, Z=5.5, p<0.001)$. The reactivity patterns of $R$. aegyptiacus sera were similar in all locations where bats of this species were sampled (Fig. 1). Further, the same pattern was observed for sera of other pteropodid bats that neutralized both SHIBV and LBV (e.g., Eidolon helvum and Epomophorus spp.; Table 1), wherein LBV-neutralizing titers were greater than SHIBV-neutralizing titers. The single exception was a subadult $R$. aegyptiacus \#593 (Fig. 2), captured in the cave where SHIBV was isolated in 2009. Serum of this bat demonstrated greater neutralizing titer against SHIBV than against LBV.

Table 2. Difference in the Seroprevalence to Shimoni Bat Virus and Lagos Bat Virus Between Hipposideros COMMERSONI AND ROUSETTUS AEGYPTIACUS

\begin{tabular}{lcc}
\hline Seroprevalence & SHIBV positive & SHIBV negative \\
\hline H. commersoni & & \\
LBV positive & 5 & 79 \\
LBV negative & 15 & \\
R. aegyptiacus & & \\
LBV positive & 26 & 42 \\
LBV negative & 0 & 42 \\
\hline
\end{tabular}

${ }^{\mathrm{a}}$ Chi-square $=20.80 ; p=0.0000$.

${ }^{\mathrm{b}}$ Chi-square $=43.99 ; p=0.000$. 

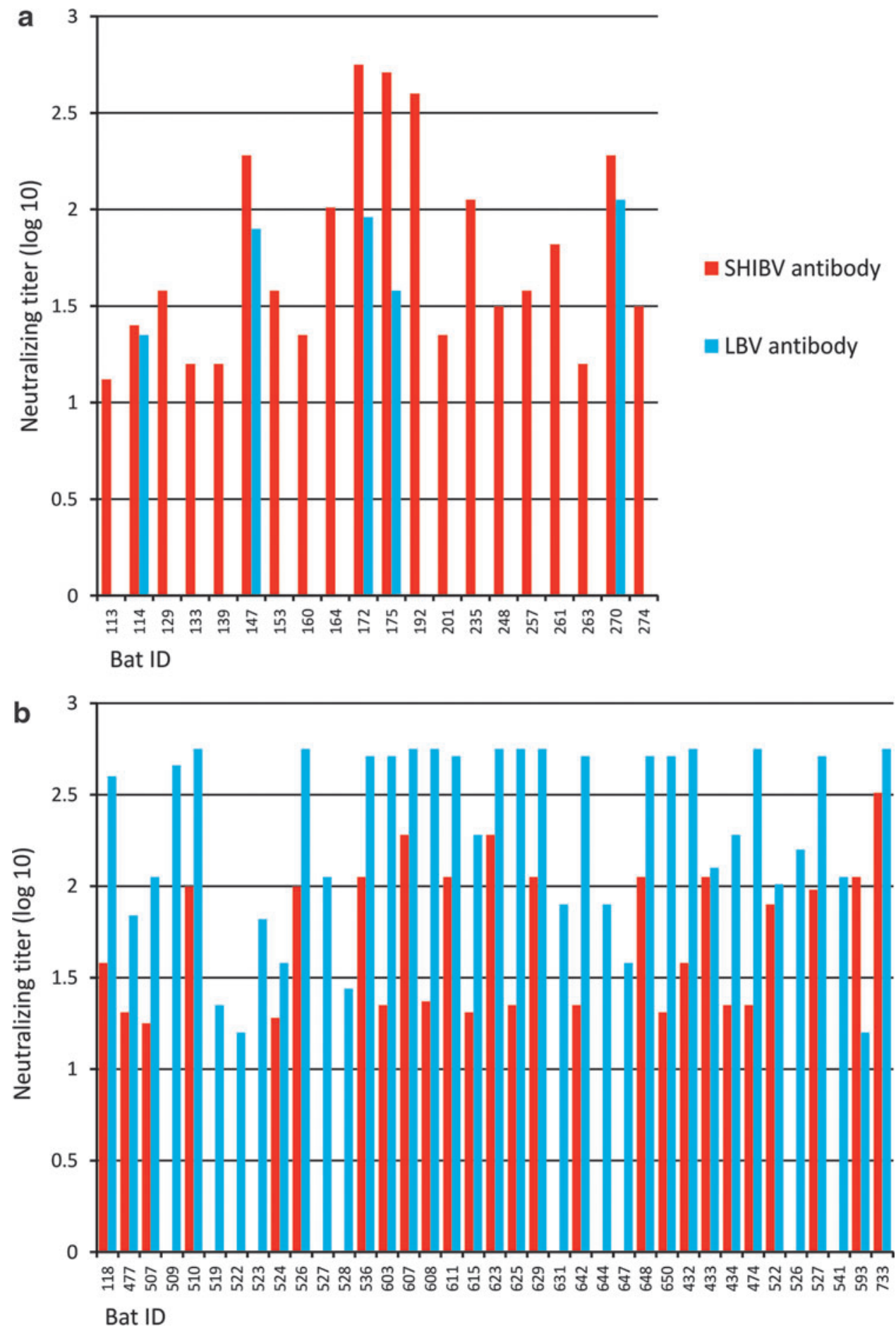

FIG. 2. Antibody titers against LBV and SHIBV in H. commersoni (a) and R. aegyptiacus (b). SHIBV, Shimoni bat virus; LBV, Lagos bat virus.

The seroprevalence of $H$. commersoni to SHIBV in Cave A (where H. commersoni roosts with bats of at least seven other species, including $R$. aegyptiacus) and in Cave B (where $H$. commersoni roosts with a limited number of insectivorous bats, but $R$. aegyptiacus is not present) was similar: $18.9 \%$ and $25.0 \%$, respectively (no statistical difference). In addition, a limited degree of serologic reactivity to SHIBV and LBV was documented in one Hipposideros spp. (presumably H. caffer or H. ruber) captured in a distant location, in two Miniopterus spp. bats, and in one Pipistrellus sp. bat (Table 1). For comparison, DUVV was not neutralized by any samples that neutralized SHIBV and LBV. In contrast, several serum samples from other bat species that neutralized DUVV did not neutralize SHIBV and LBV, suggesting specificity of 
virus neutralization in our tests, based on phylogroup difference.

\section{Discussion}

Our study demonstrates the ability to discriminate circulation of two related viruses, such as SHIBV and LBV, despite a moderate amount of antibody cross-reactivity and the absence of new isolates. Fruit bats (Family Pteropodidae) have been recognized hosts of LBV across sub-Saharan Africa since 1956. Suggested reservoir species of LBV include E. helvum (documented in Senegal, Nigeria, and Kenya), Epomophorus wahlbergi (South Africa), Micropteropus pussilus (although only one case reported from the Central African Republic), and R. aegyptiacus (Kenya and Togo or Egypt, from a case imported into France) (Aubert 1999, Kuzmin et al. 2008a, Markotter et al. 2008). Only one LBV case, considered as a spill-over, was documented in insectivorous bat Nycteris gambiensis as well as a limited number of spill-over infections in mammalian carnivores, such as cats, a dog, and a mongoose (Markotter et al. 2006, 2008). Our previous survey in Kenya demonstrated the presence of LBV and LBV-neutralizing antibodies only in fruit bats (E. helvum, R. aegyptiacus). However, only six H. commersoni were tested during that study, and all were seronegative to LBV (Kuzmin et al. 2008a).

In this survey, samples of $H$. commersoni demonstrated significantly greater seroprevalence and antibody titers to SHIBV than to LBV. More importantly, bats in Cave B had an even greater seroprevalence than bats in Cave A, suggesting intraspecific exposure to SHIBV, rather than exposure from $R$. aegyptiacus. In contrast, $R$. aegyptiacus demonstrated significantly greater seroprevalence and antibody titers to LBV than to SHIBV. Taken together, our results suggest that $H$. commersoni is the reservoir of SHIBV, at least in coastal Kenya.

The present study has several limitations. We focused primarily on $H$. commersoni and $R$. aegyptiacus and did not obtain equally representative samples from all bat species in Kenya. However, the species representation in this study (Table 1), taken in conjunction with the partial cross-reactivity between SHIBV and LBV, and the absence of LBV-seropositive bats of other species during our previous study (Kuzmin et al. 2008a) suggest that this limitation is not a significant bias. Moreover, we previously demonstrated that bats of different species apparently do not easily expose each other to lyssaviruses even when sharing roosts (Kuzmin et al. 2008b). Of course, we still cannot rule out such opportunity, particularly for the bats that demonstrated serologic reactivity to both SHIBV and LBV. However, even if such exposures occasionally occur, they did not seem to alter our results, as reactivity patterns of the samples from $H$. commersoni and $R$. aegyptiacus against SHIBV and LBV were consistently identical from caves inhabited by both species and from caves inhabited by one of these species only. The single exception is a subadult $R$. aegyptiacus that had a greater antibody titer against SHIBV than against LBV. Potentially, a cross-species exposure may explain a few cases of serologic reactivity with SHIBV and LBV detected in other bat species, such as Miniopterus sp., Pipistrellus sp., and a small-bodied Hipposideros sp. (Table 1).

Another limitation of this study is that we were unable to collect $H$. commersoni bats from various locations across the country to compare seroprevalence in distant populations.
However, we have conducted such a comparative survey for R. aegyptiacus and obtained identical serologic reactivity patterns across several locations. One additional limitation was the absence of standard immunoglobulins against SHIBV and LBV, which would facilitate better comparative quantification of the results, with expression of antibody titers in universal units. Nevertheless, the variability of viral doses in our RFFIT was insignificant, and therefore, we do not expect that it would bias results of the comparisons.

Further surveillance is needed in other populations of $H$. commersoni (including sex and age seroprevalence and longitudinal studies) to evaluate the distribution, circulation patterns, and ecology of SHIBV. Although the burden of phylogroup II lyssaviruses for veterinary and public health is unknown, this lack of knowledge is primarily based on the absence of an adequate surveillance system in the majority of African countries (Markotter et al. 2008). Initially, phylogroup II lyssaviruses were suggested to possess limited peripheral pathogenicity (Badrane et al. 2001), but these earlier experiments were performed using only one isolate of MOKV and LBV and were restricted to a murine model. Additional research demonstrated that at least some isolates of LBV are as pathogenic for mice as RABV (Markotter et al. 2009). Indeed, these viruses circulate in nature, and spill-over infections have been sporadically documented in various mammals, demonstrating the breadth of their pathogenicity. In addition, LBV and MOKV were pathogenic for nonhuman primates by peripheral routes (Tignor et al. 1973), and their significance for public health should not be underestimated. Additional concern appears from the fact that commercially available rabies biologics do not protect against phylogroup II lyssaviruses and WCBV (Bahloul et al. 1998, Jallet et al. 1999, Nel et al. 2003, Hanlon et al. 2005). Awareness of both the general public and healthcare providers regarding the potential transmission of rabies from bats must be increased, surveillance must be enhanced, and new potent biologics need to be developed to provide adequate protection against all lyssaviruses.

\section{Acknowledgments}

This study was supported in part by the Global Disease Detection Program of the Centers for Disease Control and Prevention (Atlanta, GA) and Global Disease Detection Division of CDC-Kenya (Nairobi). The authors express thanks to Edwin Danga, Evelyne Mulama, Solomon Gikundi, Leonard Nderitu, and Erick Ogola (CDC-Kenya) for excellent logistic and laboratory support and to Anne E. Mayer (University of Minnesota) for excellent technical work in the field during bat sampling. The authors appreciate discussions on bat taxonomy with Jakob Fahr (University of Ulm, Germany).

\section{Disclaimer}

Use of trade names and commercial sources are for identification only and do not imply endorsement by the U.S. Department of Health and Human Services. The findings and conclusions in this report are those of the authors and do not necessarily represent the views of the funding agency.

\section{Disclosure Statement}

No competing financial interests exist. 


\section{References}

Aubert, MFA. Rabies in individual countries. France. Rabies Bull Eur 1999; 23:6.

Badrane, H, Bahloul, C, Perrin, P, Tordo, N. Evidence of two lyssavirus phylogroups with distinct pathogenicity and immunogenicity. J Virol 2001; 75:3268-3276.

Bahloul, C, Jacob, Y, Tordo, N, Perrin, P. DNA-based immunization for exploring the enlargement of immunological cross-reactivity against the lyssaviruses. Vaccine 1998; 16: 417-425.

Dietzgen, RG, Calisher, CH, Kurath, G, Kuzmin, IV, et al. Family Rhabdoviridae. In: King, AMQ, Adams, MJ, Carstens, EB, Lefkowitz, EJ, eds. Virus Taxonomy: Ninth Report of the International Committee on Taxonomy of Viruses. London: Elsevier, 2011 (In press).

Hanlon, CA, Kuzmin, IV, Blanton, JD, Weldon, WC, et al. Efficacy of rabies biologics against new lyssaviruses from Eurasia. Virus Res 2005; 111:44-54.

Jallet, C, Jacob, Y, Bahloul, C, Drings, A, et al. Chimeric lyssavirus glycoproteins with increased immunological potential. J Virol 1999; 73:225-233.

Kingdon, J. East African Mammals. An Atlas of Evolution in Africa, Vol II, Part A (Insectivores and Bats). London-New York: Academic Press, 1974:1-341.

Kuzmin, IV, Mayer, AE, Niezgoda, M, Markotter, et al. Shimoni bat virus, a new representative of the Lyssavirus genus. Virus Res 2010; 149:197-210.

Kuzmin, IV, Niezgoda, M, Franka, R, Agwanda, B, et al. Lagos bat virus in Kenya. J Clin Microbiol 2008a; 46:14511461.

Kuzmin, IV, Niezgoda, M, Franka, R, Agwanda, B, et al. Possible emergence of West Caucasian bat virus in Africa. Emerg Infect Dis 2008b; 14:1887-1889.

Markotter, W, Kuzmin, I, Rupprecht, CE, Randles, J, et al. Isolation of Lagos bat virus from water mongoose. Emerg Infect Dis 2006; 12:1913-1918.
Markotter, W, Kuzmin, IV, Rupprecht, CE, Nel, LH. Lagos bat virus virulence in mice inoculated by the peripheral route. Epidemiol Infect 2009; 137:1155-1162.

Markotter, W, Van Eeden, C, Kuzmin, IV, Rupprecht, CE, et al. Epidemiology and pathogenicity of African bat lyssaviruses. Dev Biol (Basel) 2008; 131:317-325.

McWilliam, AN. Adaptive Responses to Seasonality in Four Species of Microchiroptera in Coastal Kenya. Ph.D. Thesis, Aberdeen: University of Aberdeen, 1982

Nel, LH, Niezgoda, M, Hanlon, CA, Morril, PA, et al. A comparison of DNA vaccines for the rabies-related virus, Mokola. Vaccine 2003; 21:2598-2606.

Simmons, NB. Order Chiroptera. In: Wilson, DE, Reeder, DM, eds. Mammal Species of the World: A Taxonomic and Geographic Reference, Vol. 1. Baltimore: John Hopkins University Press, 2005:312-529.

Smith, JS, Yager, PA, Baer, GM. A rapid fluorescent focus inhibition test (RFFIT) for determining rabies virus-neutralizing antibody. In: Meslin, F-X, Kaplan, MM, Koprowski, H, eds Laboratory Techniques in Rabies, 4th edition. Geneva: World Health Organization, 1996:181-192.

Tignor, GH, Shope, RE, Bhatt, PN, Percy, DH. Experimental infection of dogs and monkeys with two rabies serogroup viruses, Lagos bat and Mokola (IbAn 27377): clinical, serologic, virologic and fluorescent-antibody studies. J Infect Dis 1973; 128:471-478.

Address correspondence to: Ivan V. Kuzmin Rabies Program Poxvirus and Rabies Branch Division of High-Consequence Pathogens and Pathology Centers for Disease Control and Prevention 1600 Clifton Rd. Atlanta, GA 30333

E-mail: ibk3@cdc.gov 